\title{
Expression of the myosin light chains 1, 2 and 3 in the muscle of blackspot seabream (Pagellus bogaraveo, Brunnich), during development
}

\author{
P. Silva • D. M. Power $\cdot$ L. M. P. Valente $\cdot$ \\ N. Silva $\cdot$ R. A. F. Monteiro $\cdot$ E. Rocha
}

Received: 18 June 2009/Accepted: 1 March 2010/Published online: 18 March 2010

(C) Springer Science+Business Media B.V. 2010

\begin{abstract}
Previous studies on the histochemistry and immunoreactivity of fibres in lateral muscle of blackspot seabream indicated that there is a developmental transition in the composition of myofibrillar proteins, which presumably reflects changes in contractile function as the fish grows. We hypothesize that the phenomenon underscores age and spatial differences in the expression of myosin light chains (MLC), not studied yet in this species. In this study, we examined selected stages in the post-hatching development of the muscle of blackspot seabream: hatching (0 days), mouth opening (5 days), weaning (40 days) and juveniles (70 days). The spatial expression of embryonic MLC 1 (MLC1), 2 (MLC2) and 3 (MLC3) was studied by in situ hybridization. Overall,
\end{abstract}

P. Silva · L. M. P. Valente · R. A. F. Monteiro ·

E. Rocha $(\square)$

ICBAS-Institute of Biomedical Sciences Abel Salazar, Largo Prof. Abel Salazar 2, 4099-003 Porto, Portugal

e-mail: erocha@icbas.up.pt

D. M. Power · N. Silva

CCMAR-Marine Science Research Institute, CIMAR

LA - Centre for Marine and Environmental Research Associate Laboratory, University of Algarve, Campus de Gambelas, 8005-139 Faro, Portugal

P. Silva - L. M. P. Valente - R. A. F. Monteiro - E. Rocha CIIMAR - Interdisciplinary Centre for Marine and Environmental Research, CIMAR LA - Centre for Marine and Environmental Research Associate Laboratory, UPorto-University of Porto, Porto, Portugal
MLC expression patterns were overlapping and restricted to the fast muscle. At hatching and mouth opening, all MLC types were highly expressed throughout the musculature in fast muscle. The expression levels in fast muscle remained high until weaning when germinal zones appeared on the dorsal and ventral areas. The germinal zones were characterized by small-diameter fast fibres with high levels of MLC expression. This pattern persisted up to day 70, when the germinal zones disappeared and expression of MLCs was observed only in the smaller cells of the fast muscle mosaic. These results support our hypothesis and, together with previous imunoand histochemistry results, allow a better understanding of the mechanism of muscle differentiation and growth in fish beyond larval stages, and form- the basis for further comparative and experimental studies with this economically relevant species.

Keywords Blackspot seabream -

Myosin light chain - Pagellus bogaraveo .

White muscle

\section{Introduction}

In most fish, the lateral musculature is the principal propulsive organ generating motion. It differs from the skeletal muscle of other vertebrates in its 
segmental antero-posterior myotomal organization and by the fact that the two main types of fibres (fast and slow) are not intermingled but develop in physically distinct areas. The superficial red-slow fibres are responsible for slow swimming or cruising, whereas the bulk of deep white-fast fibres are used in fast burst locomotion (Johnston et al. 1977; Bone 1978). Another type of fibre, the so-called intermediate fibre, also occurs in most fish (Johnston et al. 1977). Also, fish muscle growth differs from that of mammals or birds as new fibres are continuously produced over much of the life cycle (Rowlerson and Veggetti 2001).

Myosin is essential for muscle contraction and comprises six polypeptides encoded by 3-5 genes for heavy (MHC) and light chain myosins (MLC). The relative content of MHC and MLC in muscle determines its contractile properties, including its ATPase activity, maximum shortening speeds and force-velocity relationship. The myosin complex is made of two heavy chains each of which consists of a structural $\alpha$-helical rod portion and a globular head. The myosin head contains one essential light chain and one regulatory light chain that are encoded by different genes (reviewed by Johnston and Hall 2004). In fast skeletal muscle of carp (Cyprinus carpio, Linnaeus), Hirayama et al. (1997) found that the two isoforms of essential light chain are encoded by distinct genes.

In common with terrestrial vertebrates, distinct isoforms of the myofibrillar proteins, chiefly myosin, have been described in white-fast and red-slow fish muscle fibres, with great variability between taxa (Focant et al. 1976; Rowlerson et al. 1985; Karasinski and Kilarski 1989; Devoto et al. 1996). In fish, the components of the myosin complex differ between slow and fast muscle, for example different forms of MHC are found in the two muscle types (Scapolo et al. 1988; Karasinski and Kilarski 1989; Martinez et al. 1990) and there are two types of MLC in slow muscles, and three types in fast muscle (Rowlerson et al. 1985; Karasinski and Kilarski 1989; Martinez et al. 1990).

The fibre type composition of individual muscles has been shown to vary dramatically during fish ontogeny. Various biochemical, histochemical and immunohistochemical investigations have revealed that during fish muscle development there is a sequential expression of a range of myofibrillar protein isoforms (e.g. Carpenè and Veggetti 1981; Scapolo et al. 1988; Martinez et al. 1991; Brooks and Johnston 1993; Crockford and Johnston 1993; Veggetti et al. 1993; Johnston 1994; Johnston and Horne 1994; Johnston et al. 1998; Huriaux et al. 1999, 2003; Focant et al. 2000; Moutou et al. 2001; Chauvigné et al. 2006). In the plaice Pleuronectes platessa (Linneaus), larval MLC2 is gradually replaced by adult MLC2 in postmetamorphic fish, whereas changes in myosin heavy-chain composition occurs later (Brooks and Johnston 1993). In developing turbot Scophthalmus maximus (Linneaus), sequential expression of different MHC, MLC2, troponin-T, and troponin-I isoforms occurs (Focant et al. 2000). More recently, molecular biology tools have been applied to follow the expression of isoforms at the mRNA level, offering more and new insights about muscle fibre composition in the course of fish development (Chauvigné et al. 2006; Nihei et al. 2006).

In a previous imunohistochemical study with blackspot seabream (Pagellus bogaraveo, Brunnich), a species recently adopted for aquaculture, it was shown that changes in fibre phenotype reflect developmental events in lateral muscle of this fish (Silva et al. 2008). We hypothesize that the phenomenon underscores age and spatial differences in the expression of myosin light chains. So, to further characterise the modification in myosin during the development of myotomal fibres in this species in situ hybridization was used herein to characterise the spatial and temporal expression of MLCs 1 (MLC1), 2 (MLC2) and 3 (MLC3) gene expression. This is the first study using this approach in blackspot seabream and key specific morphological and physiological development stages were chosen to investigate whether the changes in myosin are related to some precise step(s) in ontogenesis.

\section{Materials and methods}

\section{Sample collection and preparation}

Blackspot seabream were collected at $0,5,40$, and 70 days post-hatch, fixed overnight at $4{ }^{\circ} \mathrm{C}$ in $4 \%$ PFA solution (4\% paraformaldehyde in phosphate buffer at $\mathrm{pH}$ 7.4), washed three times in buffer and stored in $70 \%$ ethanol at $4{ }^{\circ} \mathrm{C}$. Samples were decalcified before further processing by immersion in $0.5 \mathrm{M}$ EDTA, $\mathrm{pH}$ 
8.0, for at least 7 days in a dark chamber. Subsequently, samples were dehydrated and wax embedded, and the serial sections $(5 \mu \mathrm{m})$ mounted on 3-aminopropyltriethoxysilane (APES)-coated slides.

\section{Histology}

\section{Probe generation}

Digoxigenin-labeled antisense RNA probes were synthesized using a digoxigenin RNA Labeling Kit (SP6/T7) (Roche Diagnostics). Linearised minipreps of pGEM-T Easy (Promega) plasmid vector containing inserts of the complete coding region of MLC1, MLC2 and MLC3, isolated from gilthead seabream (Sparus aurata, Linnaeus) were used as templates (Moutou et al. 2001). The linearised vector was purified and an in vitro transcription carried out using 20U of T7 RNA polymerase in transcription buffer (Amersham Biosciences) with $1 \mu \mathrm{l}$ of digoxigeninRNA labelling mix (Roche Diagnostics, Mannheim, Germany), for $1.5 \mathrm{~h}$ at $37^{\circ} \mathrm{C}$. The reaction was stopped with $2 \mu \mathrm{l}$ of $0.2 \mathrm{M}$ EDTA. The riboprobe was purified by lithium precipitation and resuspended in $25 \mu \mathrm{l}$ of water.

\section{Hybridisation procedure}

Sections were dewaxed in xylene and rehydrated through a graded alcohol series (100-50\%), washed twice in Tris buffered saline containing $0.1 \%$ tween20 (TBST) and pre-hybridised at $58^{\circ} \mathrm{C}$ for 120 $240 \mathrm{~min}$ in hybridisation solution $50 \%$ formamide, $4 \times \mathrm{SSC}, 0.1 \%$ torula RNA, $0.01 \%$ Heparin, $1 \times$ Denhart's, $0.1 \%$ Tween 20, 0.04\% CHAPS). Sections were then hybridised overnight at $58^{\circ} \mathrm{C}$, in hybridisation solution containing $5 \mu \mathrm{ml}^{-1}$ of riboprobe. To remove nonspecifically bound probe, stringency washes were carried out at $58^{\circ} \mathrm{C}$ first with $2 \times \mathrm{SSC}$ $(2 \times 5 \mathrm{~min})$ and then $5 \mathrm{~min}$ with $1 \times \mathrm{SSC}$. Detection of hybridised probe was carried out using antidigoxigenin-AP Fab fragments (Roche Diagnostics) (1:500) for $120 \mathrm{~min}$ at room temperature, after first blocking for $120 \mathrm{~min}$ in a $2 \%$ solution of blocking reagent (Boehringer-Mannheim) containing 10\% sheep serum. Sections were washed in two changes of TBST and colour detection was carried out at $37^{\circ} \mathrm{C}$ from 120 to $300 \mathrm{~min}$, using the chromagens NBT (4-Nitroblue tetrazolium chloride) and BCIP
(5-Bromo-4-Chloro 3-indolylphosphate). The staining reaction was stopped by washing sections in $1 \times$ PBS (Phosphate Buffered Saline) and sections were further fixed for $15 \mathrm{~min}$ with $4 \%$ paraformaldehyde at room temperature, rinsed with $1 \times$ PBS and distilled water and then mounted in glycerol gelatin.

Control experiments were performed confirming a lack of signal when samples were treated with RNAase prior to hybridisation with the riboprobe and/or by omitting riboprobe from the reaction. In order to help to detect the tissue structures some sections were stained with haematoxylin-eosin. Sections were analysed using a microscope (Olympus $\mathrm{BH} 2)$.

\section{Results and discussion}

Previously, we used histochemistry and immunohistochemistry to characterize the muscle differentiation and growth in the blackspot seabream (Silva et al. 2008). The development of lateral muscle fibre phenotypes was then described, especially their myosin isoforms, from hatching to juveniles and young adults. In this study, we used in situ hybridization for MLC to offer new insights about muscle fibre heterogeneity and functional specialization in blackspot seabream. Thus, we were able to identify fibre types as they appeared during the post-embryonic growth. Related to this we saw a high degree of similarity between intermediate and fast fibre MLC isoforms, suggesting that intermediate fibres have the same origin as fast fibres, which differs from what was previously proposed in gilthead seabream (Mascarello et al. 1995). Accordingly, the new information we obtained is important not only to tackle our questions for this species but to highlight interspecies differences, namely because muscle development from hatching to juveniles is documented for very few aquaculture fish.

The high degree of similarity among the MLC clones isolated from the gilthead seabream (Moutou et al. 2001) with the ones previously isolated from other fish allow us to conclude that the MLC expression obtained in blackspot seabream is specific. For example, the MLC1, MLC2 and MLC3 clones showed a degree of similarity with zebrafish (Danio rerio, Hamilton) of 83, 92 and 91\%, respectively. Overall, herein MLC expression was restricted to the 
fast muscle, which agrees with data obtained in juvenile and developing gilthead seabream (Moutou et al. 2001). In common with the gilthead seabream, a notable change in the tissue expression pattern of the various forms of MLC occurs during blackspot seabream development. This may be a general feature of MLCs during fish development, as changes in myosin light chains have also been observed in an immunohistochemical study of Atlantic herring ( $\mathrm{Clu}$ pea harengus, Linnaeus) at different development stages (Crockford and Johnston 1993).

At hatching, MLCs were uniformly expressed in the deep fast muscle fibres of blackspot seabream. This spatial distribution of fast muscle isoform transcripts is consistent with the previous identification at hatching of two major fibre types [superficial monolayer (LSM) and fast muscle (LW)] in that species (Silva et al. 2008), as shown by immunohistochemistry using anti-myosin sera. This result suggests that the superficial monolayer fibres contain a specific myosin isoform which is an agreement with our previous immunohistochemical results (Silva et al. 2008). The MLC disposition in early developing blackspot seabream muscle, however, differs from rainbow trout (Oncorhynchus mykiss, Walbaum) larvae (Chauvigné et al. 2006), in which several fast isoform mRNAs, including MLC1 and MLC3, selectively accumulate in the superficial neoformed slow muscle. In blackspot seabream at 5 days of age (mouth opening, i.e. transition from endogenous to exogenous feeding), the in situ hybridization result was the same as the one obtained at hatching (Fig. 1). The small group of slow fibres adjacent to the lateral line nerve identified for the first time at this age by imunohistochemistry (Silva et al. 2008) contains none of the three MLC transcripts studied.

At 40 days of larval life (weaning, i.e. transition from live to formula food), a strong MLC signal was detected at the periphery of the dorsal and ventral germinal zones (Fig. 2). The germinal zones were characterized by small-diameter fast fibres with high levels of MLC expression, whereas the deeper layer consisted of polygonal. This result suggests that MLC transcripts are largely restricted to myoblasts and/or young fibres that arise in those germinal zones, thus making MLC a useful marker of hyperplasia during this stratified growth phase. Previous work based on BrdU-labeling and ultrastructural observation has shown that, during larval life, differentiation of slow and fast fibre types is supported by a hyperplastic mechanism that occurs by apposition of new fibres along proliferating zones, principally under the lateral line and in the apical myomere regions, and also just under the superficial monolayer (Rowlerson et al. 1995). With the ultimate aim of examining the sites and the timing of myogenic activation in pearlfish (Rutilus meidingery, Heckel) embryos and larvae, Steinbacher and co-authors (2006) studied the role of myocyte-specific enhancer factor 2 (MEF2) transcription factors, which are known to bind the promoter and enhancer regions of teleost Myogenin and MLC genes. They observed that in germinal zones, most MEF2+ cells give rise to fast fibres and that only a few such cells generate new slow fibres at the terminations of the superficial slow muscle layer, which is in agreement with our results.

Also at 40 days of life, an additional fibre type was identified by immunohistochemistry (Silva et al.
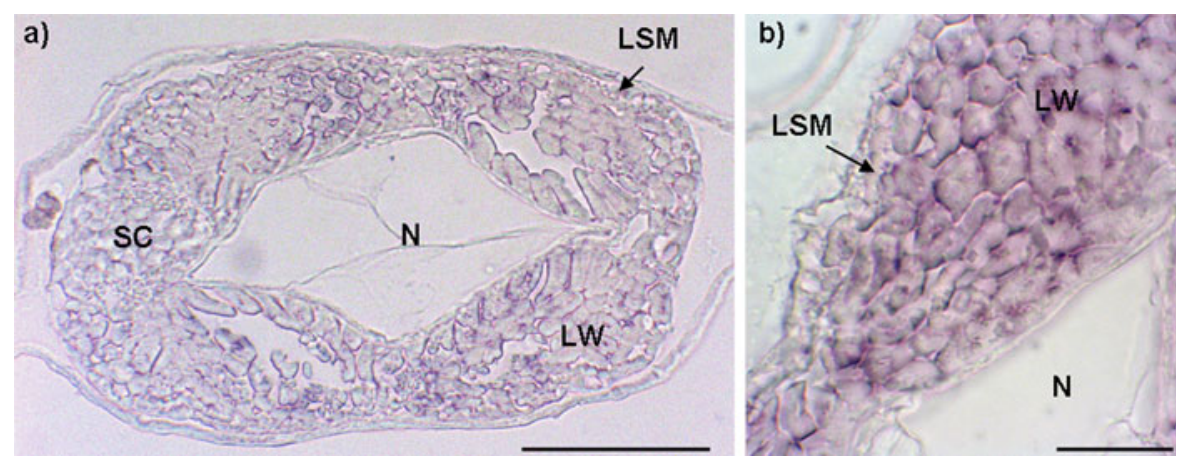

Fig. 1 Temporal and spatial expression of MLC1 in transverse sections of the posterior lateral muscle of Pagellus bogaraveo aged 5 days. The larvae white-fast muscle (LW) showed MLC1 expression. Other symbols: SC, spinal cord, $N$, notochord, LSM, superficial monolayer. Scale bar $=25 \mu \mathrm{m}$ 

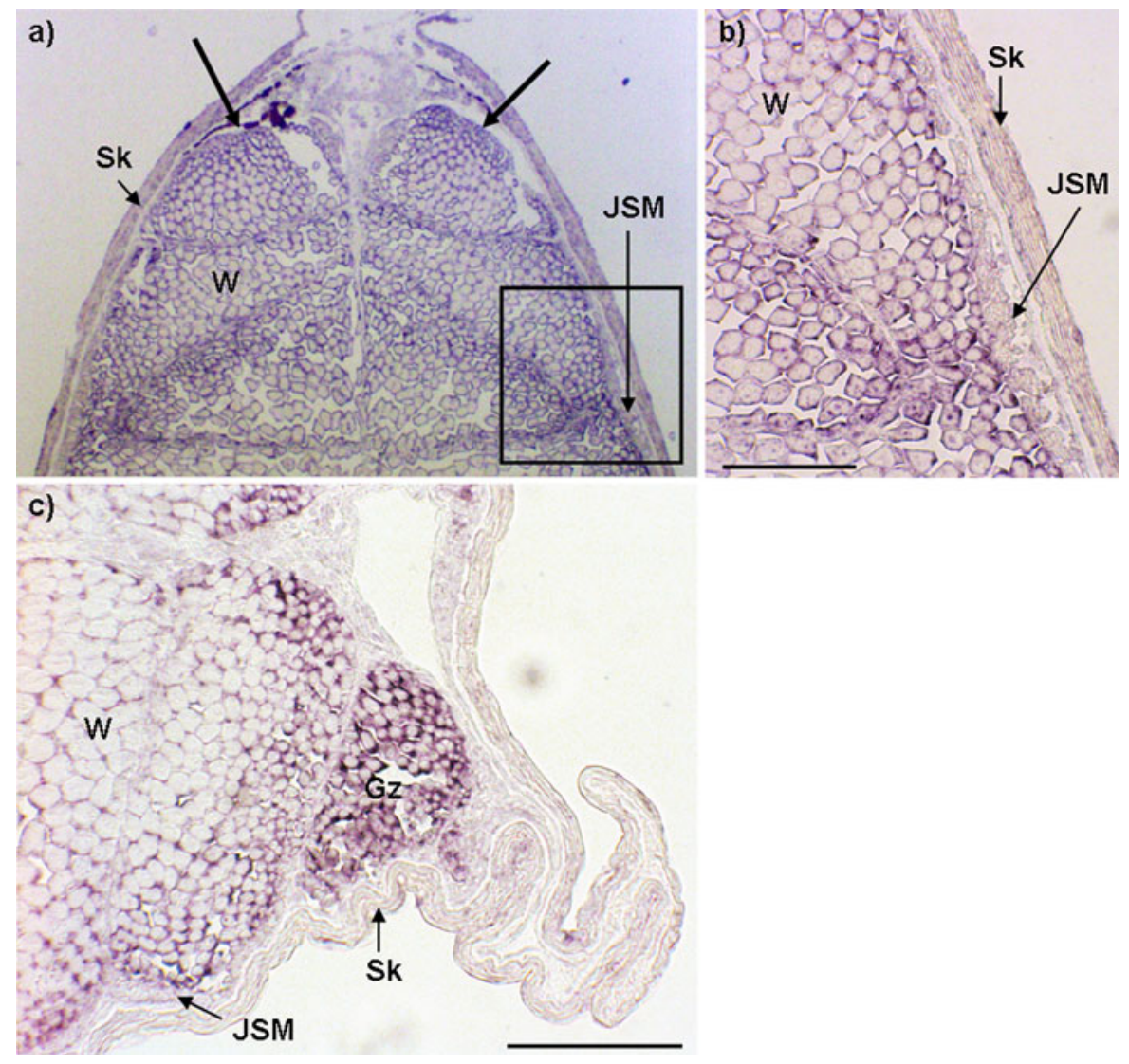

Fig. 2 In situ hybridization showing MLC3 expression in white-fast muscle of Pagellus bogaraveo aged 40 days. Note the lateral germinal zones-thicker arrows in (a) and $\mathrm{Gz}$ in (c). Boxed area in (a) is shown at higher magnification on the right

2008), forming a distinctive 'pink' or intermediate layer. In gilthead seabream, another sparid fish, the same germinal zone under the lateral line that gives rise to additional slow fibres from hatching to the midpoint of larval life also gives rise to intermediate (pink) fibres (Mascarello et al. 1995). In blackspot seabream, however, the new muscle cells arising at the lateral growth zones are MLC + which suggests that the MLC gene(s) expressed by intermediate fibres diverge only slight from fast fibres in their nucleotide sequence, thus causing cross-reaction of the fast MLC probe. It remains unknown at this point whether this difference between these two sparid fish has a functional meaning. Eventually the reassessment using molecular techniques could elucidate the pink-intermediate muscle fibre origin in this important fish group.

In blackspot seabream of 70 days, several myosepta separating adjacent $\mathrm{W}$-shaped myotomes are (b). The juvenile superficial monolayer (JSM) showed no MLC3 expression. Other symbols: $W$, adult fast white muscle; Sk, skin. Scale bar $=25 \mu \mathrm{m}$

visible in the transverse sections (Fig. 3). The expression of MLC was limited to the small diameter fast muscle fibres (W) which appeared not only near to myosepta but also dispersed in the fast muscle at 70 days post hatching, i.e. in early juvenile life, and this was reflected by a wide range of fibre sizes that gave the mosaic appearance in cross section (Fig. 3). The mosaic character of muscle has been suggested to be a result of hyperplastic processes generally only occurring in fish which grow to a large final size (Carpenè and Veggetti 1981; Rowlerson and Veggetti 2001; Sänger and Stoiber 2001; Johnston 2006). The small diameter fibres in the fast muscle of blackspot seabream also differed from the large diameter fibres in their ATPase reactivity (Silva et al. 2008). It has been suggested that the de novo myogenesis process which occurs in growing muscle is similar to that observed during embryonic development (Johnston 


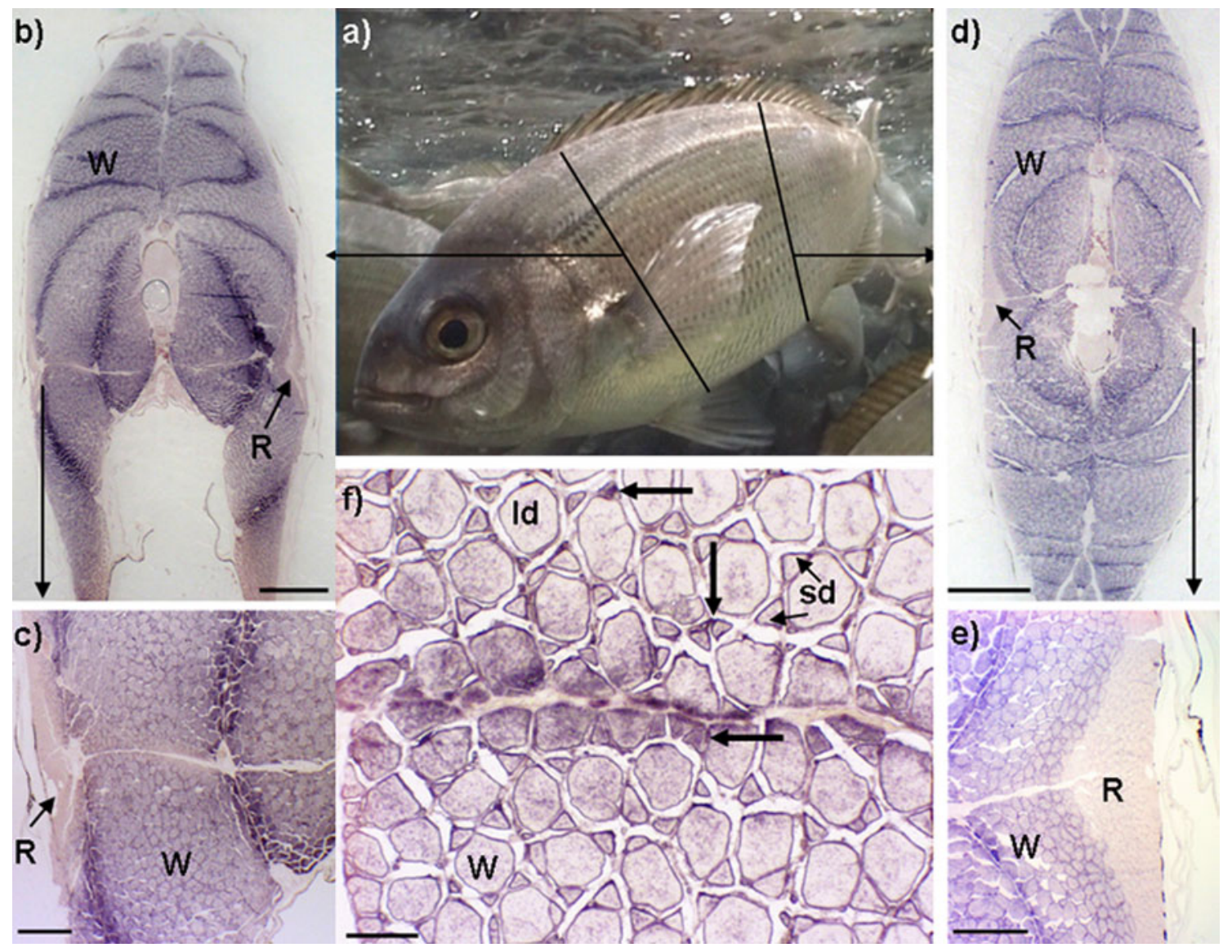

Fig. 3 Transverse section of lateral muscle of Pagellus bogaraveo (a) aged 70 days showing MLC1 expression at post-opercular level (b and c) and at post-anal level (d, e and f). f shows the "mosaic appearance" of the white-fast muscle. Thicker arrows indicate cells in the inter-fibre space expressing

1999). The persistence of early MLC isoforms in growing blackspot seabream could be related to this process. As mention above, an accumulation of MLC transcripts were noted near to myosepta which is consistent with the suggestion by Stoiber and Sänger (1996) that the mosaic precursors migrate on a longer route, probably using myoseptal gaps to reach their final position between the pre-established fibres.

In conclusion, the observations support our hypothesis that transition in the composition of myofibrillar proteins in blackspot seabream, from hatching to juveniles, is founded in age and spatial differences in the expression of myosin light chains. We saw that the temporal and spatial MLC expression patterns follow fast muscle development and mark the germinal zones and the newly formed fast
MLC1. Symbols: ld, large-diameter white-fast fibres; $s d$, smalldiameter white-fast fibres; $W$, adult white-fast muscle; $R$, adult red-slow fibres. Scale bar $(\mathbf{b}),(\mathbf{d})=250 \mu \mathrm{m} ;(\mathbf{c}),(\mathbf{e})=100 \mu \mathrm{m}$ and $(\mathbf{f})=25 \mu \mathrm{m}$

fibres, indicating their embryonic character. The changes in myofibrillar gene transcripts and probable protein composition during the early life of blackspot seabream observed in this study probably reflect a modification in the functional properties of the muscle as body size increases. The fibre type transitions described in both this and in a previous paper (Silva et al. 2008) demonstrate that gene expression of this post-mitotic cell can be altered with age. Future research will be needed to elucidate in more detail the molecular elements and mechanisms underlying fibre type determination and transformation in blackspot seabream.

Acknowledgments Work partially supported by FCT (Foundation for Science and Technology) PhD Grant SFRH-BD-14068- 
2003 attributed to P. Silva. The authors are greatly indebted to the "Instituto Español de Oceanografía" (Centro Oceanográfico de Vigo, España) which provided the fish.

\section{References}

Bone Q (1978) Locomotor muscle. In: Hoar WS, Randall DJ (eds) Fish physiology, vol VII. Academic Press, New York, pp 361-424

Brooks S, Johnston IA (1993) Influence of development and rearing temperature on the distribution, ultrastructure and myosin sub-unit composition of myotomal muscle-fibre types in the plaice Pleuronectes platessa. Mar Biol 117:501-513

Carpenè E, Veggetti A (1981) Increase in muscle fibers in the lateralis muscle (white portion) of Mugilidae (Pisces, Teleostei). Experientia 37:191-193

Chauvigné F, Ralliere C, Cauty C, Rescan PY (2006) In situ hybridisation of a large repertoire of muscle-specific transcripts in fish larvae: the new superficial slow-twitch fibres exhibit characteristics of fast-twitch differentiation. J Exp Biol 209:372-379

Crockford T, Johnston IA (1993) Development changes in the composition of myofibrillar proteins in the swimming muscles of Atlantic herring, Clupea harengus. Mar Biol 115:15-22

Devoto SH, Melancon E, Eisen JS, Westerfield M (1996) Identification of separate slow and fast muscle precursor cells in vivo, prior to somite formation. Development $122: 3371-3380$

Focant B, Huriaux F, Johnston IA (1976) Subunit composition of fish myofibrils: the light chains of myosin. Int J Biochem 7:129-133

Focant B, Collin S, Vandewalle P, Huriaux F (2000) Expression of myofibrillar proteins and parvalbumin isoforms in white muscle of the developing turbot Scophthalmus maximus (Pisces, Pleuronectiformes). Basic Appl Myol 10:269-278

Hirayama Y, Kanoh S, Nakaya M, Watabe S (1997) The two essential light chains of carp fast skeletal myosin, LC1 and LC3, are encoded by distinct genes and change their molar ratio following temperature acclimation. J Exp Biol 200:693-701

Huriaux F, Vandewalle P, Baras E, Legendre M, Focant B (1999) Myofibrillar proteins in white muscle of the developing African catfish Heterobranchus longifili ( $\mathrm{Si}$ luriforms, Clariidae). Fish Physiol Biochem 21:287-301

Huriaux F, Baras E, Vandewalle P, Focant B (2003) Expression of myofibrillar proteins and parvalbumin isoforms in white muscle of dorada during development. J Fish Biol 62:774-792

Johnston IA (1994) Development and plasticity of fish muscle with growth. Basic Appl Myol 4:353-368

Johnston IA (1999) Muscle development and growth: potential implications for flesh quality in fish. Aquaculture 177: 99-115

Johnston IA (2006) Environment and plasticity of myogenesis in teleost fish. J Exp Biol 209:2249-2264

Johnston IA, Hall TE (2004) Mechanisms of muscle development and responses to temperature change in fish larvae.
In: Govoni JJ (ed) The development of form and function in fish and the question of larval adaptation, American fisheries society symposium, 40. Bethesda, Maryland, pp 113-144

Johnston IA, Horne Z (1994) Immunocytochemical investigations of muscle differentiation in the Atlantic herring (Clupea harengus: teleostei). J Mar Biol Assoc UK 74: 79-91

Johnston IA, Davison W, Goldspink G (1977) Energy metabolism of carp swimming muscles. J Comp Physiol 114: 203-216

Johnston IA, Cole NJ, Abercromby M, Vieira VLA (1998) Embryonic temperature modulates muscle growth characteristics in larval and juvenile herring. J Exp Biol 201:623-646

Karasinski J, Kilarski W (1989) Polymorphism of myosin isoenzymes and myosin heavy chains in histochemically typed skeletal muscles of the roach (Rutilus rutilus L., Cyprinidae, Fish). Comp Biochem Physiol 92B:727-731

Martinez I, Ofstad R, Olsen RL (1990) Intraspecific myosin light chain polymorphism in the white muscle of herring (Clupea harengus harengus, L.). FEBS Lett 265(1, 2):2326

Martinez I, Christiansen JS, Ofstad R, Olsen RL (1991) Comparison of myosin isoenzymes present in skeletal and cardiac muscles of the Arctic charr Salvelinus alpinus (L.): sequential expression of different myosin heavy chains during development of the fast white muscle. Eur J Biochem 195:743-753

Mascarello F, Rowlerson A, Radaelli P, Veggetti A (1995) Differentiation and growth of muscle in the fish Sparus aurata (L): I. Myosin expression and organization of fibre types in lateral muscle from hatching to adult. J Muscle Res Cell Mot 16:213-222

Moutou KA, Canario AVM, Marmuris Z, Power DM (2001) Molecular cloning and sequence of Sparus aurata skeletal myosin light chains expressed in white muscle: developmental expression and thyroid regulation. J Exp Biol 204:3009-3018

Nihei Y, Kobiyama A, Ikeda D, Ono Y, Ohara S, Cole NJ, Johnston IA, Watabe S (2006) Molecular cloning and mRNA expression analysis of carp embryonic, slow and cardiac myosin heavy chain isoforms. J Exp Biol 209: 188-198

Rowlerson A, Veggetti A (2001) A Cellular mechanisms of post-embryonic muscle growth in aquaculture species. In: Johnston IA (ed) Muscle development and growth. Fish physiology series vol 18. Academic Press, San Diego, pp 103-140

Rowlerson A, Scapolo PA, Mascarello F, Carpenè E, Veggetti A (1985) Comparative study of myosin present in lateral muscle of some fish: species variations in myosin isoforms and their distribution in red-slow, pink and white muscle. J Muscle Res Cell Mot 6:601-640

Rowlerson A, Mascarello F, Radaelli G, Veggetti A (1995) Differentiation and growth of muscle in the fish Sparus aurata (L): II. Hyperplastic and hypertrophic growth of lateral muscle from hatching to adult. J Muscle Res Cell Mot 16:223-236

Sänger AM, Stoiber W (2001) Muscle fiber diversity and plasticity. In: Johnston IA (ed) Muscle development and 
growth. Fish physiology series vol 18. Academic Press, San Diego, pp 187-250

Scapolo PA, Veggetti A, Mascarello F, Romanello MG (1988) Development transitions of myosin isoforms and organisation of the lateral muscle in the teleost Dicentrarchus labrax (L.). Anat Embryol 178:287-295

Silva P, Rowlerson AM, Valente LMP, Olmedo M, Monteiro RAF, Rocha E (2008) Muscle differentiation and growth in blackspot seabream (Pagellus bogaraveo, Brunnich): histochemical and immunohistochemical study of the fibre types. Tissue Cell 40:447-458

Steinbacher P, Haslett JR, Six M, Gollmann HP, Sänger AM, Stoiber W (2006) Phases of myogenic cell activation and possible role of dermomyotome cells in teleost muscle formation. Dev Dynam 235:3132-3143

Stoiber W, Sänger AM (1996) An electron microscopic investigation into possible source of new muscle fibres in teleost fish. Anat Embryol 194:569-579

Veggetti A, Mascarello F, Scapolo PA, Rowlerson A, Carnevali CMD (1993) Muscle growth and myosin isoform transitions during development of a small teleost fish, Poecilia reticulata (Peters) (Atheriniformes, Poeciliidae): a histochemical, immunohistochemical, ultrastructural and morphometric study. Anat Embryol 187:353-361 